\title{
Diagnostic Value of Subjective Memory Complaints Assessed with a Single Item in Dominantly Inherited Alzheimer's Disease: Results of the DIAN Study
}

\author{
Christoph Laske, ${ }^{1,2,3}$ Hamid R. Sohrabi, ${ }^{4,5}$ Mateusz S. Jasielec, ${ }^{6}$ Stephan Müller, ${ }^{3}$ \\ Niklas K. Koehler, ${ }^{3}$ Susanne Gräber, ${ }^{1,7}$ Stefan Förster, ${ }^{8}$ Alexander Drzezga, \\ Felix Mueller-Sarnowski, ${ }^{10,11}$ Adrian Danek, ${ }^{10,11}$ Mathias Jucker, ${ }^{1,12}$ Randall J. Bateman, ${ }^{13}$ \\ Virginia Buckles, ${ }^{13}$ Andrew J. Saykin, ${ }^{14}$ Ralph N. Martins, ${ }^{4,5}$ John C. Morris, ${ }^{13}$ \\ and Dominantly Inherited Alzheimer Network (DIAN) ${ }^{15}$ \\ ${ }^{1}$ German Center for Neurodegenerative Diseases (DZNE), 72076 Tübingen, Germany \\ ${ }^{2}$ Section for Dementia Research, Department of Cellular Neurology, Hertie Institute for Clinical Brain Research and \\ Department of Psychiatry and Psychotherapy, University of Tübingen, 72076 Tübingen, Germany \\ ${ }^{3}$ Department of Psychiatry and Psychotherapy, University of Tübingen, 72076 Tübingen, Germany \\ ${ }^{4}$ Centre of Excellence for Alzheimer's Disease Research and Care, School of Medical Sciences, Edith Cowan University, \\ Perth, WA 6027, Australia \\ ${ }^{5}$ School of Psychiatry and Clinical Neurosciences, University of Western Australia, Nedlands, WA 6009, Australia \\ ${ }^{6}$ Division of Biostatistics, Washington University School of Medicine, St. Louis, MO 63108, USA \\ ${ }^{7}$ Department of Neurodegeneration, Hertie Institute for Clinical Brain Research, University of Tübingen, 72076 Tübingen, Germany \\ ${ }^{8}$ Department of Nuclear Medicine \& TUM Neuroimaging Center (TUM-NIC), Klinikum Rechts der Isar, \\ Technical University Munich, 80333 Munich, Germany \\ ${ }^{9}$ Department of Nuclear Medicine, University of Cologne, 50937 Cologne, Germany \\ ${ }^{10}$ Deutsches Zentrum für Neurodegenerative Erkrankungen, 81377 München, Germany \\ ${ }^{11}$ Neurologische Klinik und Poliklinik, Ludwig-Maximilians-Universität, 81377 München, Germany \\ ${ }^{12}$ Department of Cellular Neurology, Hertie Institute for Clinical Brain Research, University of Tübingen, 72076 Tübingen, Germany \\ ${ }^{13}$ Department of Neurology, Knight Alzheimer's Disease Research Center, Washington University School of Medicine, \\ St. Louis, MO 63108, USA \\ ${ }^{14}$ Indiana Alzheimer Disease Center, Indiana University School of Medicine, Indianapolis, IN 46202, USA \\ ${ }^{15}$ Department of Neurology, Washington University in St. Louis, MO 63108, USA
}

Correspondence should be addressed to Christoph Laske; christoph.laske@med.uni-tuebingen.de

Received 7 November 2014; Accepted 11 March 2015

Academic Editor: Annalena Venneri

Copyright (C) 2015 Christoph Laske et al. This is an open access article distributed under the Creative Commons Attribution License, which permits unrestricted use, distribution, and reproduction in any medium, provided the original work is properly cited.

Objective. We examined the diagnostic value of subjective memory complaints (SMCs) assessed with a single item in a large crosssectional cohort consisting of families with autosomal dominant Alzheimer's disease (ADAD) participating in the Dominantly Inherited Alzheimer Network (DIAN). Methods. The baseline sample of 183 mutation carriers (MCs) and 117 noncarriers (NCs) was divided according to Clinical Dementia Rating (CDR) scale into preclinical (CDR 0; MCs: $n=107$; NCs: $n=109$ ), early symptomatic (CDR 0.5; MCs: $n=48$; NCs: $n=8$ ), and dementia stage (CDR $\geq 1$; MCs: $n=28$; NCs: $n=0$ ). These groups were subdivided by the presence or absence of SMCs. Results. At CDR 0, SMCs were present in $12.1 \%$ of MCs and 9.2\% of NCs $(P=0.6)$. At CDR 0.5, SMCs were present in $66.7 \%$ of MCs and $62.5 \%$ of NCs $(P=1.0)$. At CDR $\geq 1$, SMCs were present in $96.4 \%$ of MCs. SMCs in MCs were significantly associated with CDR, logical memory scores, Geriatric Depression Scale, education, and estimated years to onset. Conclusions. The present study shows that SMCs assessed by a single-item scale have no diagnostic value to identify preclinical ADAD in asymptomatic individuals. These results demonstrate the need of further improvement of SMC measures that should be examined in large clinical trials. 


\section{Introduction}

Alzheimer's disease (AD) is currently conceptualized as progressing in three stages including (1) preclinical AD, (2) early symptomatic AD that has been characterized as mild cognitive impairment (MCI) due to $\mathrm{AD}$, and (3) dementia due to $\mathrm{AD}$ [1]. After publication of these research criteria in 2011, the research focus has moved to the preclinical stage, as it is considered a highly promising target for future early intervention [2]. Thus, identification of early symptoms characterizing the preclinical stage of AD is highly needed.

Although interest is increasing in subjective memory complaints (SMCs), it remains uncertain due to controversial findings whether they are meaningful or not with respect to the diagnosis of preclinical sporadic AD. Some studies have found that SMCs are associated with an increased risk of developing $\mathrm{AD}[3,4]$, while other studies have found that they are not predictive of cognitive impairment or future cognitive decline [5-7] and may correlate better with depressive symptoms than with cognitive symptoms [8-11]. The terms "subjective memory/cognitive complaints/decline" have been used in different ways throughout the last 40 years. In earlier years, "subjective" meant "reporting memory concerns by the subject in any stage of cognitive impairment" [12-16]. In most current literature, the term "subjective cognitive decline" is used for defining a state in the preclinical stage of sporadic $\mathrm{AD}$ and "subjective" means "in absence of objective impairment" [17]. In the present study, we use the term SMC because individuals are asked concerning their memory decline and not concerning other cognitive functions. In principle, the term SMC can be used at different stages of cognitive impairment, as done in the present study, as far as in which stage it is examined is defined (e.g., "SMC in preclinical AD," "SMC in MCI," or "SMC in dementia").

Autosomal dominant $\mathrm{AD}$ (ADAD) is a rare form of $\mathrm{AD}$, caused by mutations in genes encoding presenilin-1 (PSEN1), presenilin-2 (PSEN2), or amyloid precursor protein (APP) and leading to young onset dementia. The opportunity to observe trajectories of changes in biological and psychological parameters of dementia-related processes is explored by the Dominantly Inherited Alzheimer Network (DIAN), studying ADAD in a large sample of pedigrees with early onset forms of dementia. Baseline characteristics and biomarker findings in this cohort are on record [18, 19]. A previous study with carriers of the PSEN1 E280A mutation identified a pre-MCI stage with already existing memory complaints [20]. However, this study focused on mutation carriers (MCs) and did not describe the frequency of SMCs in noncarriers (NCs) and the diagnostic value of SMCs in the different stages of ADAD.

At present the diagnostic value of SMCs in the different stages of ADAD is unclear. The aim of the current study was to address this open question by examining the frequency and diagnostic accuracy of SMCs in MCs and NCs of ADAD participating in the DIAN study in different clinical stages.

\section{Materials and Methods}

All aspects of the study were approved by the institutional review boards for each of the participating sites in DIAN. All participants provided written informed consent.

2.1. Participants. The baseline sample of $183 \mathrm{MCs}$ and $117 \mathrm{NCs}$ participants (first-degree relatives of MCs) of the DIAN study underwent clinical assessment of cognitive status by means of the Clinical Dementia Rating (CDR) scale [21, 22] and was consecutively divided into preclinical (asymptomatic; CDR 0 ), early symptomatic (CDR 0.5$)$, and dementia $(\mathrm{CDR} \geq 1)$ groups. Only MCs but not NCs fulfilled the criterion of CDR $\geq 1$. Thus, $\mathrm{CDR} \geq 0.5$ refers to the combination of CDR groups 0.5 and higher for the MCs compared to the CDR 0.5 NCs. Tables 1 and 2 show their demographic data. SMCs were assessed on Form B9 (Clinician Judgment of Symptoms: "Does the subject report a decline in memory?") of the National Alzheimer's Coordinating Center (NACC) Uniform Data Set (UDS) [23]. The groups were subdivided by the presence or absence of SMCs. Estimated years to onset (EYO) were calculated as the age of the participant at assessment minus the age of the parent at symptom onset. EYO was given minus values in case of the years before estimated disease onset and plus values in case of the years after estimated disease onset. As all participants of the DIAN study are members of affected ADAD families, the construct of EYO can be applied to all (MCs and NCs), resulting in age-matched cases and controls. This construct of EYO has been validated in the DIAN study as providing a highly predictable clinical estimate of stage of $\mathrm{AD}$ biomarker profiles and symptom onset [18]. When the study was started, approximately $85 \%$ of participants did not know their mutation status. This recently has changed over the past one to two years with more participants having genetic counseling and testing. We estimated up to $40 \%$ now knowing their genetic status. However, the information which participant knows the mutation status is not available in the present database.

2.2. Measurement Methods. All demographical (age, gender, education, and estimated years to onset [EYO]), clinical (Geriatric Depression Scale [GDS]), and cognitive measurements (e.g., CDR global scores and sum of boxes [21, 22], logical memory subtest of The Wechsler Memory Test) were performed as recently described $[18,19]$.

2.3. Data Analysis. Subject characteristics were summarized as mean and standard deviation (SD) for continuous variables or number for categorical variables. Comparisons between the MCs and NCs on these characteristics were made with independent Mann-Whitney $U$ tests or Chi-squared tests, respectively, Fisher's exact test in case of small expected cell counts (less than 5). The analyses of the overall association between memory complaints and CDR groups were performed using the Cochran-Armitage exact trend test, with Cramer's $V$ used to gauge the relative magnitude of the association. For determination of diagnostic accuracy, we calculated the following characteristic numbers: accuracy, 
TABLE 1: Characteristics of the participants of the DIAN study.

\begin{tabular}{lccc}
\hline Variables & $\begin{array}{c}\text { Mutation carriers (MCs) } \\
(N=183)\end{array}$ & $\begin{array}{c}\text { Noncarriers (NCs) } \\
(N=117)\end{array}$ & 3 value \\
\hline Age (years) & $39.1 \pm 10.2$ & $47 / 70.10 .3$ & $0.6771^{*}$ \\
Gender (M/F) & $81 / 102$ & $14.6 \pm 2.7$ & $0.485^{* *}$ \\
Education level (years) & $13.9 \pm 2.7$ & $0.03 \pm 0.1$ & $0.0343^{*}$ \\
CDR global & $0.3 \pm 0.6$ & $0.1 \pm 0.2$ & $<0.0001^{*}$ \\
CDR sum of boxes & $1.64 \pm 3.2$ & 8 & $<0.0001^{*}$ \\
CDR 0 (number) & 107 & 0 & $<92^{* *}$ \\
CDR 0.5 (number) & 48 & $60.9 \pm 31.8$ & $<.0001^{* *}$ \\
CDR $\geq 1$ (number) & 28 & $29.03 \pm 1.3$ & $<$. \\
Logical memory, delayed recall (percentile) & $38.5 \pm 36.8$ & $1.3 \pm 1.6$ & $<0.0001^{*}$ \\
MMSE & $26.8 \pm 7.5$ & $<0.0001^{*}$ \\
GDS & $3.8 \pm 11.2$ & $<0.0001^{*}$ \\
\hline
\end{tabular}

${ }^{*}$ Mann-Whitney $U$ test; ${ }^{* *}$ Chi-square test; CDR denotes Clinical Dementia Rating Scale; M denotes male and F denotes female; MMSE denotes Mini-Mental Status Examination; GDS denotes Geriatric Depression Scale; age, education levels, CDR sum of boxes, MMSE, logical memory, and GDS scores are displayed in mean and standard deviation.

sensitivity, specificity, positive predictive value (PPV), and negative predictive value (NPV). Significance for the results was set at $P<0.05$. All statistical analyses were performed using SAS 9.3 (SAS Institute, Inc., Cary, NC).

\section{Results}

3.1. Demographics of Participants of the DIAN Study. MCs and NCs were comparable regarding age and gender, showing no significant differences (Table 1). In addition, MCs had significantly lower educational levels, MMSE, and logical memory scores and higher CDR global scores, CDR sum of boxes, and GDS scores than their sibling NCs (Table 1). Table 2 shows the characteristics of the participants of the DIAN study by CDR stage and carrier status. A comparison of the characteristics of the noncarriers (NCs) with CDR 0 versus CDR 0.5 is displayed in Table 3.

3.2. Percentage and Diagnostic Accuracy of SMCs in Participants of the DIAN Study Depending on Clinical Stage. At CDR 0 , SMCs were present in $12.1 \%$ of MCs and $9.2 \%$ of NCs (Chisquare test: $P=0.478$ ). At CDR 0.5 , SMCs were present in $66.7 \%$ of MCs and $62.5 \%$ of NCs (Fisher's exact test: $P=1.0$ ). At $C D R \geq 1$, SMCs were present in $96.4 \%$ of MCs (Figure 1).

Diagnostic accuracy of SMCs for being a MC at CDR 0 was $51.9 \%$ (sensitivity $12.2 \%$, specificity $90.8 \%$, PPV $56.5 \%$, and NPV 51.3\%), at CDR $0.562 .5 \%$ (sensitivity 66.7\%, specificity $37.5 \%$, PPV $86.5 \%$, and NPV $15.8 \%$ ), and at CDR $\geq 0.5$ (referring to the combination of CDR groups 0.5 and higher for the MCs compared to the CDR $0.5 \mathrm{NCs}) 73.8 \%$ (sensitivity $77.6 \%$, specificity $37.5 \%$, PPV $92.2 \%$, and NPV $15.0 \%)$.

\subsection{Association between SMCs and Clinical Parameters in Par-} ticipants of the DIAN Study. SMCs were positively correlated with CDR stages in MCs (Cramer's $V=0.687$; trend test $P<0.0001$ ) and in NCs (Cramer's $V=0.403$; trend test $P=$

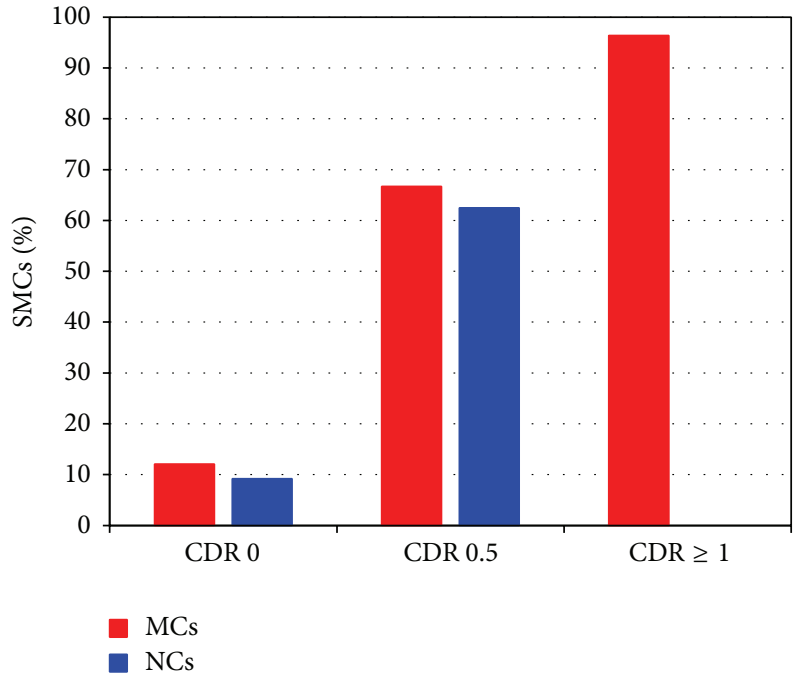

FIGURE 1: Percentage of mutation carriers (MCs) and noncarriers (NCs) with subjective memory complaints (SMCs) as a function of Clinical Dementia Rating (CDR) scale.

$0.0008)$. SMCs were inversely correlated with logical memory scores in MCs (Cramer's $V=-0.541$; trend test $P<0.0001$ ) but not in NCs (Cramer's $V=-0.154$; trend test $P=0.098$ ). In addition, SMCs were positively correlated with EYO in MCs (Cramer's $V=0.609$; trend test $P<0.0001$ ), meaning that MCs with EYO $<0$ who were closer to onset (less years to onset) or MCs with $\mathrm{EYO} \geq 0$ who were farther from onset had a higher likelihood of SMCs but not in NCs (Cramer's $V=0.370$; trend test $P=0.5809$ ). Furthermore, SMCs were significantly inversely related to education in the cohort as a whole (Cramer's $V=-0.175$; trend test $P=0.0005$ ) and in MCs (Cramer's $V=-0.1352$; trend test $P=0.0248$ ) and NCs (Cramer's $V=-0.2241$; trend test $P=0.0341$ ) separately. Moreover, SMCs were positively correlated with GDS scores 
TABLE 2: Characteristics of the participants of the DIAN study by CDR stage and carrier status.

\begin{tabular}{|c|c|c|c|}
\hline Variables & $\begin{array}{c}\text { Mutation carriers (MCs) } \\
(N=183)\end{array}$ & $\begin{array}{c}\text { Noncarriers (NCs) } \\
(N=117)\end{array}$ & $P$ value \\
\hline \multicolumn{4}{|c|}{ CDR 0} \\
\hline Age (years) & $35.1 \pm 8.8$ & $39.4 \pm 10.2$ & $0.002^{*}$ \\
\hline Gender (M/F) & $46 / 61$ & $45 / 64$ & $0.800^{* *}$ \\
\hline Education level (years) & $14.2 \pm 2.7$ & $14.7 \pm 2.7$ & $0.092^{*}$ \\
\hline CDR sum of boxes & $0.03 \pm 0.1$ & $0.01 \pm 0.08$ & $0.186^{*}$ \\
\hline Logical memory, delayed recall (percentile) & $55.3 \pm 33.2$ & $62.2 \pm 31.1$ & $0.181^{*}$ \\
\hline MMSE & $29.0 \pm 1.3$ & $29.1 \pm 1.3$ & $0.488^{*}$ \\
\hline GDS & $1.6 \pm 1.9$ & $1.2 \pm 1.5$ & $0.129^{*}$ \\
\hline SMCs (number) & 13 & 10 & $0.478^{* *}$ \\
\hline \multicolumn{4}{|c|}{ CDR 0.5} \\
\hline Age (years) & $42.6 \pm 9.3$ & $42.4 \pm 12.4$ & $0.774^{*}$ \\
\hline Gender (M/F) & $21 / 27$ & $2 / 6$ & $0.449^{* * *}$ \\
\hline Education level (years) & $13.4 \pm 2.3$ & $12.5 \pm 3.4$ & $0.50^{*}$ \\
\hline CDR sum of boxes & $1.5 \pm 1.1$ & $0.6 \pm 0.4$ & $0.007^{*}$ \\
\hline Logical memory, delayed recall (percentile) & $17.4 \pm 27.6$ & $44.8 \pm 39.4$ & $0.015^{*}$ \\
\hline MMSE & $26.6 \pm 2.8$ & $28.4 \pm 1.6$ & $0.09^{*}$ \\
\hline GDS & $3.7 \pm 3.4$ & $2.1 \pm 2.5$ & $0.198^{*}$ \\
\hline SMCs (number) & 32 & 5 & $1.000^{* * *}$ \\
\hline \multicolumn{4}{|c|}{$\mathrm{CDR} \geq 1$} \\
\hline Age (years) & $48.4 \pm 8.6$ & n.a. & \\
\hline Gender (M/F) & $14 / 14$ & 0 & \\
\hline Education level (years) & $12.8 \pm 2.7$ & n.a. & \\
\hline CDR sum of boxes & $7.9 \pm 4.1$ & n.a. & \\
\hline Logical memory, delayed recall (percentile) & $10.4 \pm 27.1$ & n.a. & \\
\hline MMSE & $15.6 \pm 6.3$ & n.a. & \\
\hline GDS & $4.1 \pm 3.1$ & n.a. & \\
\hline SMCs (number) & 27 & 0 & \\
\hline
\end{tabular}

${ }^{*}$ Mann-Whitney $U$ test; ${ }^{* *}$ Chi-square test; ${ }^{* * *}$ Fisher's exact test; CDR denotes Clinical Dementia Rating Scale; M denotes male and F denotes female; MMSE denotes Mini-Mental Status Examination; GDS denotes Geriatric Depression Scale; age, education levels, CDR sum of boxes, MMSE, logical memory, and GDS scores are displayed in mean and standard deviation.

in MCs (Cramer's $V=0.394$; trend test $P<0.0001$ ) and in NCs (Cramer's $V=0.197$; trend test $P=0.033$ ).

Using Spearman partial correlation, we found that SMCs in MCs were significantly correlated with logical memory scores even after controlling for GDS scores $(r=-0.396 ; P<$ 0.0001). In NCs, SMCs showed a trend towards significance with logical memory after controlling for GDS $(r=-0.179$; $P=0.055)$.

Using linear mixed models, MCs with SMCs showed significantly lower logical memory scores than MCs without SMCs at CDR 0.5 (Mean \pm Standard Error: $5.1 \pm 0.8$ versus 9.8 $\pm 1.1 ; P=0.001)$ and at $C D R \geq 0.5(8.3 \pm 2.8$ versus $9.2 \pm 1.2$; $P<0.0001)$ and a trend of significance at CDR $0(10.9 \pm 1.1$ versus $13.3 \pm 0.5 ; P=0.066)$. In addition, MCs with SMCs showed significantly higher GDS scores than MCs without SMCs at CDR 0.5 (Mean \pm Standard Error: $4.4 \pm 0.7$ versus $2.3 \pm 0.6 ; P=0.001)$ and at $\mathrm{CDR} \geq 0.5(8.4 \pm 2.5$ versus $2.2 \pm$ $0.5 ; P<0.0001)$ and a trend of significance at CDR $0(2.3 \pm$ 0.8 versus $1.5 \pm 0.2 ; P=0.083$ ).

\section{Discussion}

The main findings of the present study were as follows. (1) Preclinical (asymptomatic) MCs of ADAD did not more frequently report SMCs in comparison with asymptomatic NCs. (2) The percentage and diagnostic accuracy of SMCs for being a MC increased with advancing cognitive impairment. (3) In MCs, SMCs were significantly positively associated with CDR, GDS, and EYO and inversely with education and logical memory scores.

In most current literature, the term "subjective cognitive decline" is used for defining a state in the preclinical stage of sporadic $\mathrm{AD}$ without objective cognitive impairment [17]. However, in the present study, the percentage of asymptomatic individuals with SMCs was rather low $(\leq 12.1 \%)$ and did not differ between MCs and NCs. Thus, SMCs in asymptomatic members of ADAD families did not segregate with mutation status. This finding is in line with previous studies, showing that SMCs are not predictive of cognitive 
TABLE 3: Comparison of the characteristics of the noncarriers (NCs) with CDR 0 versus CDR 0.5 .

\begin{tabular}{|c|c|c|c|}
\hline Variables & $\begin{array}{c}\text { Noncarriers (NCs) CDR } 0 \\
(N=109)\end{array}$ & $\begin{array}{c}\text { Noncarriers (NCs) CDR } 0.5 \\
(N=8)\end{array}$ & $P$ value \\
\hline Age (years) & $39.4 \pm 10.2$ & $42.4 \pm 12.4$ & $0.552^{*}$ \\
\hline Gender (M/F) & $45 / 64$ & $2 / 6$ & $0.427^{* *}$ \\
\hline Education level (years) & $14.7 \pm 2.7$ & $12.5 \pm 3.4$ & $0.068^{*}$ \\
\hline CDR sum of boxes & $0.01 \pm 0.08$ & $0.6 \pm 0.4$ & $<0.0001^{*}$ \\
\hline Logical memory, delayed recall (percentile) & $62.2 \pm 31.1$ & $44.8 \pm 39.4$ & $0.257^{*}$ \\
\hline MMSE & $29.1 \pm 1.3$ & $28.4 \pm 1.6$ & $0.125^{*}$ \\
\hline GDS & $1.2 \pm 1.5$ & $2.1 \pm 2.5$ & $0.285^{*}$ \\
\hline SMCs (number) & 10 & 5 & $0.001^{* *}$ \\
\hline
\end{tabular}

${ }^{*}$ Mann-Whitney $U$ test; ${ }^{* *}$ Fisher's exact test; CDR denotes Clinical Dementia Rating Scale; M denotes male and F denotes female; MMSE denotes Mini-Mental Status Examination; GDS denotes Geriatric Depression Scale; age, education levels, CDR sum of boxes, MMSE, logical memory, and GDS scores are displayed in mean and standard deviation.

impairment or future cognitive decline [5-7]. A previous study with carriers of the PSEN1 E280A mutation identified a pre-MCI stage, characterized by memory complaints and coexisting objective cognitive impairment [20]. These findings indicate that SMCs in ADAD seem to occur rarely without additional objective cognitive impairment which is different from what is expected in sporadic AD [17]. This potential difference between ADAD and sporadic AD could be explained by a more aggressive course of ADAD [24] with an earlier onset of objective cognitive impairment and thus with a shorter asymptomatic phase.

The relevance of assessing SMCs is not restricted to preclinical $\mathrm{AD}$, as nonspecialists in primary care are inaccurate at identifying dementia as well as MCI [25]. In the present study, frequency of SMCs clearly increased during the course of ADAD along with advancing cognitive impairment. According to a meta-analysis, SMCs were reported by $38 \%$ of those with known MCI and $43 \%$ of those with known dementia [16]. The higher percentage of SMCs in individuals with early symptomatic ADAD (66.7\%) and dementia due to ADAD (96.4\%) in the present study could be explained by the fact that members of families affected with ADAD may be more aware of or vigilant towards the cognitive changes than individuals in the general population. Additionally, the ADAD individuals are usually younger than those with late onset $\mathrm{MCI} / \mathrm{AD}$ and therefore, any cognitive decline is more readily noticed without being ascribed to "age-related" limitations that can commonly be seen in older adults.

In the present study, $9.2 \%$ of asymptomatic NCs reported SMCs. This result is not surprising as in a recent study $14.4 \%$ of young adults (aged 18-39 years) reported SMCs [26]. Interestingly, 8/117 NCs showed in the present study a CDR of 0.5. Comparing the baseline characteristics in NCs with CDR 0.5 versus NCs with CDR 0 revealed that NCs with CDR 0.5 also showed higher CDR sum of boxes scores, a higher rate of SMCs, and a trend of lower education level compared to NCs with CDR 0.

Several previous studies have demonstrated a relationship between SMCs and depressive mood states [8-11]. In line with these findings, we also found positive correlations between SMCs and GDS scores in MCs and NCs. In addition, MCs with SMCs showed significantly higher GDS scores than MCs without SMCs at CDR 0.5 and at CDR $\geq 0.5$ and a trend of significance at CDR 0. However, the significant relationship between SMCs and GDS does not rule out an ongoing neurodegenerative process or cognitive deterioration. We reported a significant association between SMCs, cognitive function (as measured by the logical memory subtest of the Wechsler Memory Test), and cognitive impairments (CDR results) as well as higher memory difficulties in MCs with SMCs. Interestingly, in MCs, we reported a significant association between subjective and objective measures of memory, even after controlling for depression effects (as measured using GDS). This significant association was not observed for NCs after controlling for depression score. These findings signify the sensitivity of SMCs to cognitive changes due to neurodegenerative processes of $\mathrm{AD}$ while their specificity requires further investigations.

A limitation of the study was that SMCs were captured by use of a single question with a binary threshold based on clinician judgement, which may be not sensitive enough to detect more subtle self-perceived changes. However, using a single-item question on SMCs has been previously reported to predict late onset $\mathrm{AD}$ incidence [3]. Another limitation was that the question concerning SMCs specifically referred to memory and did not include decline in behaviour, motor, or other cognitive functions. Furthermore, the number of individuals with SMCs in the preclinical stage of $A D$ was rather small. In addition, the data analyzed were cross-sectional baseline data of the DIAN study, with limited predictive power concerning the EYO as opposed to individual longitudinal data. However, no sufficient longitudinal data are as yet available. It should also be noted that due to younger age, clinical observations resulted from studying ADAD may not be directly transferable to those in sporadic $\mathrm{AD}$ and vice versa.

\section{Conclusions}

Data of the present study indicate that SMCs assessed by a single-item scale have no diagnostic value to identify preclinical ADAD in asymptomatic individuals. These results demonstrate the need of further improvement of SMC 
assessment (at least in the ADAD population), for example, by additional use of multi-items and quantitative measures of SMCs. Additionally, multidomain cognitive complaint measures and inclusion of the informant part may increase the clinical utility of this construct. The diagnostic and clinical value of different SMC test instruments should be examined now in larger clinical trials with sporadic AD patients. The significant relationship of SMCs with logical memory and dementia stages, as we reported here, warrants further investigation into its clinical applications in general practice within elderly population.

\section{Disclosure}

Randall J. Bateman has received personal compensation for activities with Link Medicine, JAI, Bristol-Myers Squibb Company, Pfizer Inc., Merck, SPRI, Elan Corporation, Eisai Inc., and Medtronic, Inc., received royalty payments from Washington University, and received research support from Astra Zeneca Pharmaceuticals and Merck \& Co., Inc. He has received personal compensation in an editorial capacity for serving as Associate Editor, Journal of Neuropsychiatry and Clinical Neurosciences. Ralph N. Martins is the founder and owns stock in Alzhyme. Hamid R. Sohrabi has received personal compensation for activities with Pfizer and Wyeth and is the Western Australian Site Neuropsych Lead for TOMMORROW Study by the Takeda Pharmaceuticals. John C. Morris reports the following disclosure: Neither Dr. Morris nor his family owns stock or has equity interest (outside of mutual funds or other externally directed accounts) in any pharmaceutical or biotechnology company. Dr. Morris has participated or is currently participating in clinical trials of antidementia drugs sponsored by the following companies: Janssen Immunotherapy and Pfizer. Dr. Morris has served as a consultant for Lilly USA. He receives research support from Eli Lilly/Avid Radiopharmaceuticals and is funded by $\mathrm{NIH}$ Grants nos. P50AG005681, P01AG003991, P01AG026276, and U19AG032438.

\section{Conflict of Interests}

Christoph Laske, Mateusz S. Jasielec, Stephan Müller, Niklas K. Koehler, Susanne Gräber, Stefan Förster, Alexander Drzezga, Felix Mueller-Sarnowski, Adrian Danek, Mathias Jucker, Virginia Buckles, and Andrew J. Saykin report no potential conflict of interests.

\section{Acknowledgments}

This study was funded by the National Institute on Aging (DIAN; U19 AG032438; John C. Morris, PI) and by the German Center for Neurodegenerative Diseases (DZNE), Bonn, Germany. This work involved an international consortium, which included contributions by the following Site/Core leaders: Randy Bateman, Tammie Benzinger, Nigel Cairns, Anne Fagan, Bernardino Ghetti, Alison Goate, Daniel Marcus, Ralph Martins, Colin Masters, Richard Mayeux, Eric McDade, John Morris, John Ringman, Martin
Rossor, Stephen Salloway, Peter Schofield, Reisa Sperling, and Chengjie Xiong. The authors thank the DIAN participants and their families for their dedication and altruism and the research and support staff at each of the DIAN sites for their contributions to this study (http://www.dian-info.org/ personnel.htm).

\section{References}

[1] C. R. Jack Jr., M. S. Albert, D. S. Knopman et al., "Introduction to the recommendations from the National Institute on AgingAlzheimer's Association workgroups on diagnostic guidelines for Alzheimer's disease," Alzheimer's \& Dementia, vol. 7, no. 3, pp. 257-262, 2011.

[2] R. A. Sperling, P. S. Aisen, L. A. Beckett et al., "Toward defining the preclinical stages of Alzheimer's disease: recommendations from the National Institute on Aging-Alzheimer's Association workgroups on diagnostic guidelines for Alzheimer's disease," Alzheimer's and Dementia, vol. 7, no. 3, pp. 280-292, 2011.

[3] M. I. Geerlings, C. Jonker, L. M. Bouter, H. J. Adèr, and B. Schmand, "Association between memory complaints and incident Alzheimer's disease in elderly people with normal baseline cognition," The American Journal of Psychiatry, vol. 156, no. 4, pp. 531-537, 1999.

[4] F. Jessen, B. Wiese, C. Bachmann et al., "Prediction of dementia by subjective memory impairment effects of severity and temporal association with cognitive impairment," Archives of General Psychiatry, vol. 67, no. 4, pp. 414-422, 2010.

[5] D. B. Carr, S. Gray, J. Baty, and J. C. Morris, "The value of informant versus individual's complaints of memory impairment in early dementia," Neurology, vol. 55, no. 11, pp. 1724-1726, 2000.

[6] M. E. M. Mol, M. P. J. van Boxtel, D. Willems, and J. Jolles, "Do subjective memory complaints predict cognitive dysfunction over time? A six-year follow-up of the Maastricht Aging Study," International Journal of Geriatric Psychiatry, vol. 21, no. 5, pp. 432-441, 2006.

[7] S. Hollands, Y. Y. Lim, R. Buckley et al., "Amyloid-beta related memory decline is not associated with subjective or informant rated cognitive impairment in healthy adults," vol. 43 , no. 2 , pp. 677-686, 2015.

[8] Y. Balash, M. Mordechovich, H. Shabtai, D. Merims, and N. Giladi, "Subjective memory decline in healthy communitydwelling elders. What does this complain mean?" Acta Neurologica Scandinavica, vol. 121, no. 3, pp. 194-197, 2010.

[9] M. Reid, L. Parkinson, R. Gibson et al., "Memory complaint questionnaire performed poorly as screening tool: validation against psychometric tests and affective measures," Journal of Clinical Epidemiology, vol. 65, no. 2, pp. 199-205, 2012.

[10] Y. Balash, M. Mordechovich, H. Shabtai, N. Giladi, T. Gurevich, and A. D. Korczyn, "Subjective memory complaints in elders: depression, anxiety, or cognitive decline?" Acta Neurologica Scandinavica, vol. 127, no. 5, pp. 344-350, 2013.

[11] R. Buckley, M. M. Saling, D. Ames et al., "Factors affecting subjective memory complaints in the AIBL aging study: biomarkers, memory, affect, and age," International Psychogeriatrics, vol. 25, no. 8, pp. 1307-1315, 2013.

[12] B. Reisberg, S. H. Ferris, M. J. De Leon, and T. Crook, "The global deterioration scale for assessment of primary degenerative dementia," American Journal of Psychiatry, vol. 139, no. 9, pp. 1136-1139, 1982.

[13] B. Reisberg, "Dementia: a systematic approach to identifying reversible causes," Geriatrics, vol. 41, no. 4, pp. 30-46, 1986. 
[14] R. C. Petersen, G. E. Smith, S. C. Waring, R. J. Ivnik, E. G. Tangalos, and E. Kokmen, "Mild cognitive impairment: clinical characterization and outcome," Archives of Neurology, vol. 56, no. 3, pp. 303-308, 1999.

[15] M. S. Albert, S. T. DeKosky, D. Dickson et al., "The diagnosis of mild cognitive impairment due to Alzheimer's disease: recommendations from the National Institute on AgingAlzheimer's Association workgroups on diagnostic guidelines for Alzheimer's disease," Alzheimer's \& Dementia, vol. 7, no. 3, pp. 270-279, 2011.

[16] A. J. Mitchell, "The clinical significance of subjective memory complaints in the diagnosis of mild cognitive impairment and dementia: a meta-analysis," International Journal of Geriatric Psychiatry, vol. 23, no. 11, pp. 1191-1202, 2008.

[17] F. Jessen, R. E. Amariglio, M. van Boxtel et al., "A conceptual framework for research on subjective cognitive decline in preclinical Alzheimer's disease," Alzheimer's and Dementia, vol. 10, no. 6, pp. 844-852, 2014.

[18] R. J. Bateman, C. Xiong, T. L. Benzinger et al., "Clinical and biomarker changes in dominantly inherited Alzheimer's disease," The New England Journal of Medicine, vol. 367, no. 9, pp. 795-804, 2012.

[19] J. C. Morris, P. S. Aisen, R. J. Bateman et al., "Developing an international network for Alzheimer research: the dominantly inherited Alzheimer network," Clinical Investigation, vol. 2, no. 10, pp. 975-984, 2012.

[20] N. Acosta-Baena, D. Sepulveda-Falla, C. M. Lopera-Gómez et al., "Pre-dementia clinical stages in presenilin 1 E280A familial early-onset Alzheimer's disease: a retrospective cohort study," The Lancet Neurology, vol. 10, no. 3, pp. 213-220, 2011.

[21] J. C. Morris, “The Clinical Dementia Rating (CDR): current version and scoring rules," Neurology, vol. 43, no. 11, pp. 24122414, 1993.

[22] J. C. Morris, C. Ernesto, K. Schafer et al., "Clinical dementia rating training and reliability in multicenter studies: the Alzheimer's Disease Cooperative Study experience," Neurology, vol. 48, no. 6, pp. 1508-1510, 1997.

[23] J. C. Morris, S. Weintraub, H. C. Chui et al., "The Uniform Data Set (UDS): clinical and cognitive variables and descriptive data from Alzheimer disease centers," Alzheimer Disease and Associated Disorders, vol. 20, no. 4, pp. 210-216, 2006.

[24] L. Wu, P. Rosa-Neto, G.-Y. R. Hsiung et al., "Early-onset familial alzheimer's disease (EOFAD)," Canadian Journal of Neurological Sciences, vol. 39, no. 4, pp. 436-445, 2012.

[25] A. Connolly, E. Gaehl, H. Martin, J. Morris, and N. Purandare, "Underdiagnosis of dementia in primary care: variations in the observed prevalence and comparisons to the expected prevalence," Aging and Mental Health, vol. 15, no. 8, pp. 978984, 2011.

[26] S. T. Chen, P. Siddarth, L. M. Ercoli et al., "Modifiable risk factors for Alzheimer disease and subjective memory impairment across age groups," PLoS ONE, vol. 9, no. 6, Article ID e98630, 2014. 


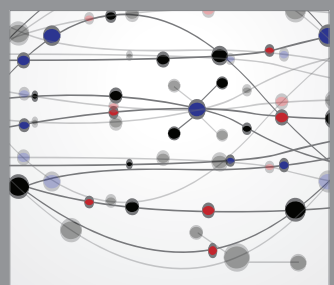

The Scientific World Journal
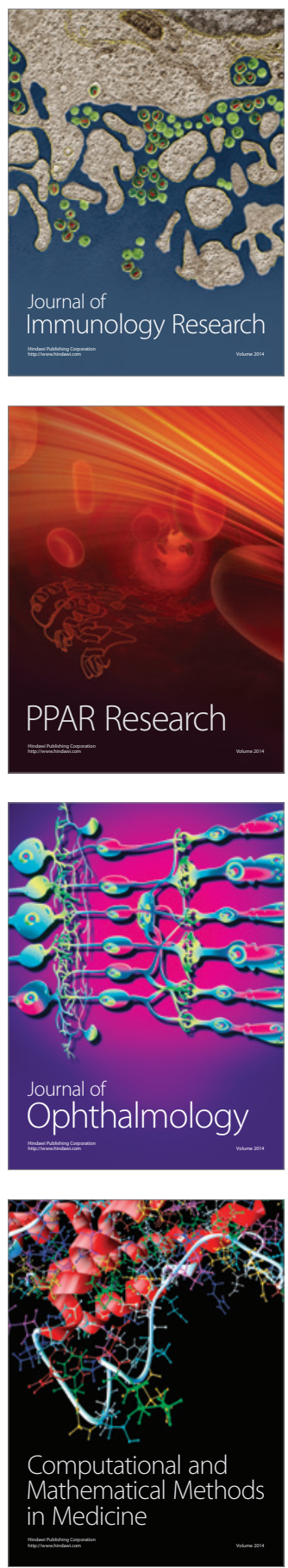

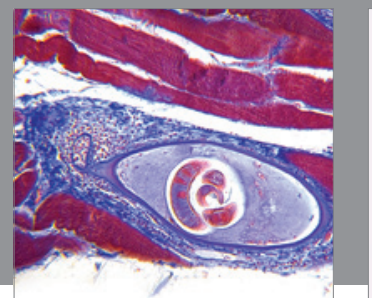

Gastroenterology

Research and Practice
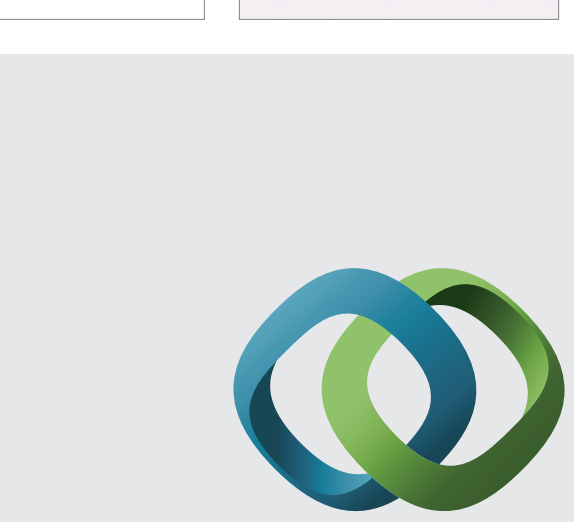

\section{Hindawi}

Submit your manuscripts at

http://www.hindawi.com
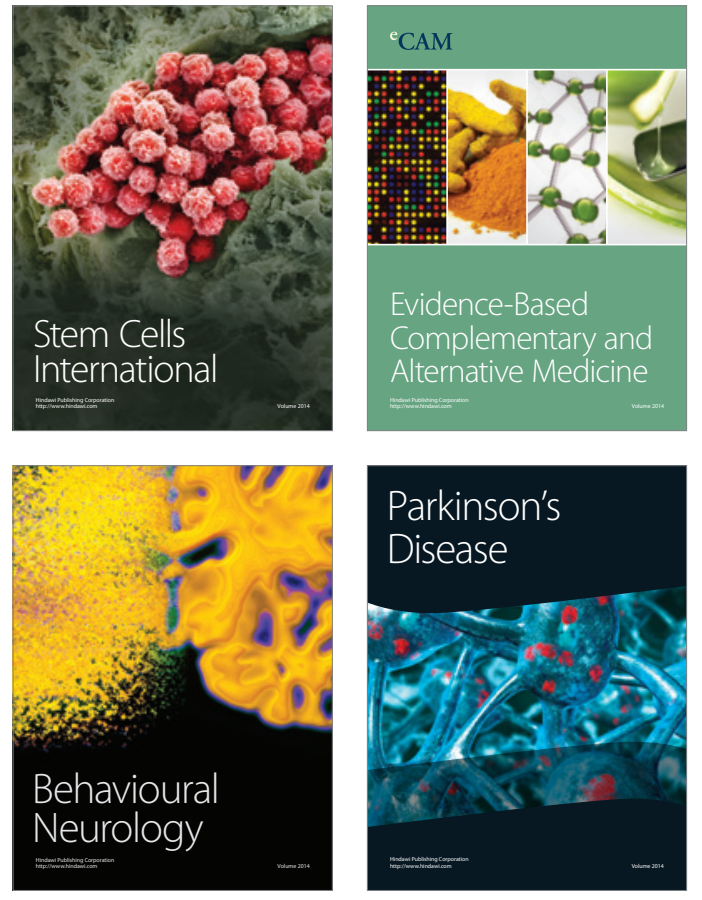
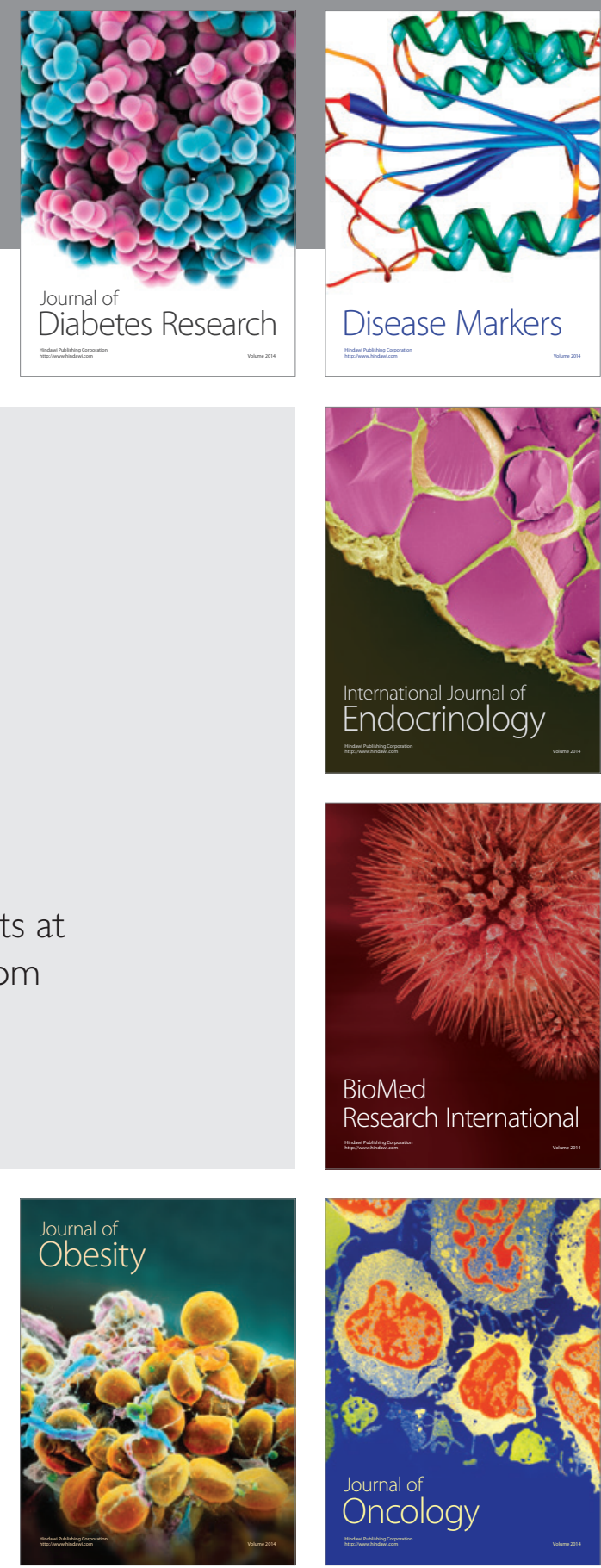

Disease Markers
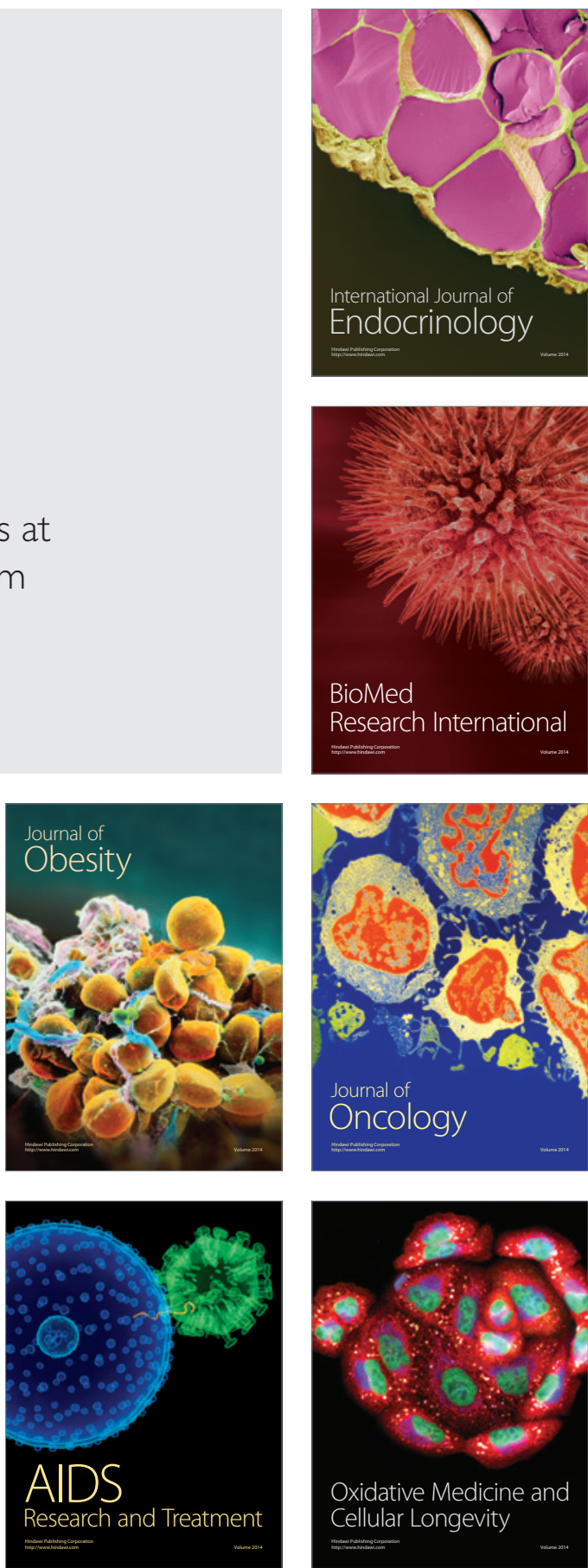